\title{
A CLASS OF HYPERGRAPHS THAT GENERALIZES CHORDAL GRAPHS
}

\author{
ERIC EMTANDER
}

\begin{abstract}
In this paper we introduce a class of hypergraphs that we call chordal. We also extend the definition of triangulated hypergraphs, given by H. T. Hà and A. Van Tuyl, so that a triangulated hypergraph, according to our definition, is a natural generalization of a chordal (rigid circuit) graph. R. Fröberg has showed that the chordal graphs corresponds to graph algebras, $R / I(\mathscr{G})$, with linear resolutions. We extend Fröberg's method and show that the hypergraph algebras of generalized chordal hypergraphs, a class of hypergraphs that includes the chordal hypergraphs, have linear resolutions. The definitions we give, yield a natural higher dimensional version of the well known flag property of simplicial complexes. We obtain what we call $d$-flag complexes.
\end{abstract}

\section{Introduction and preliminaries}

Let $\mathscr{X}$ be a finite set and $\mathscr{E}=\left\{E_{1}, \ldots, E_{s}\right\}$ a finite collection of non empty subsets of $\mathscr{X}$. The pair $\mathscr{H}=(\mathscr{X}, \mathscr{E})$ is called a hypergraph. The elements of $\mathscr{X}$ and $\mathscr{E}$, respectively, are called the vertices and the edges, respectively, of the hypergraph. If we want to specify what hypergraph we consider, we may write $\mathscr{X}(\mathscr{H})$ and $\mathscr{E}(\mathscr{H})$ for the vertices and edges respectively. A hypergraph is called simple if: (1) $\left|E_{i}\right| \geq 2$ for all $i=1, \ldots, s$ and (2) $E_{j} \subseteq E_{i}$ only if $i=j$. If the cardinality of $\mathscr{X}$ is $n$ we often just use the set $[n]=\{1,2, \ldots, n\}$ instead of $\mathscr{X}$.

Let $\mathscr{H}$ be a hypergraph. A subhypergraph $\mathscr{K}$ of $\mathscr{H}$ is a hypergraph such that $\mathscr{X}(\mathscr{K}) \subseteq \mathscr{X}(\mathscr{H})$, and $\mathscr{E}(\mathscr{K}) \subseteq \mathscr{E}(\mathscr{H})$. If $\mathscr{Y} \subseteq \mathscr{X}$, the induced hypergraph on $\mathscr{Y}, \mathscr{H}_{y}$, is the subhypergraph with $\mathscr{X}\left(\mathscr{H}_{\mathscr{Y}}\right)=\mathscr{Y}$ and with $\mathscr{E}\left(\mathscr{H}_{\mathscr{Y}}\right)$ consisting of the edges of $\mathscr{H}$ that lie entirely in $\mathscr{Y}$. A hypergraph $\mathscr{H}$ is said to be $d$ uniform if $\left|E_{i}\right|=d$ for every edge $E_{i} \in \mathscr{E}(\mathscr{H})$. By a uniform hypergraph we mean a hypergraph that is $d$-uniform for some $d$. Note that a simple 2-uniform hypergraph is just an ordinary simple graph.

Throughout the paper we denote by $R$ the polynomial ring $k\left[x_{1}, \ldots, x_{n}\right]$ over some field $k$, where $n$ is the number of vertices of a hypergraph considered at the moment. By identifying each vertex $v_{i} \in \mathscr{X}(\mathscr{H})$ with a variable $x_{i} \in R$, we may think of an edge $E_{i}$ of a hypergraph as a monomial $x^{E_{i}}=\prod_{j \in E_{i}} x_{j}$

Received 28 April 2008. 
in $R$. Employing this idea, we may associate to every simple hypergraph $\mathscr{H}$, a squarefree monomial ideal in $R$. The edge ideal $I(\mathscr{H})$ of a hypergraph $\mathscr{H}$ is the ideal

$$
I(\mathscr{H})=\left(x^{E_{i}} ; E_{i} \in \mathscr{E}(\mathscr{H})\right) \subseteq R,
$$

generated "by the edges" of $\mathscr{H}$.

The edge ideal was first introduced by R. H. Villarreal [18], in the case when $\mathscr{H}=\mathscr{G}$ is a simple graph. After that, hypergraph algebras have been widely studied. See for instance [5], [7], [10], [11], [12], [13], [14], [16], [19]. In [10], the authors use certain connectedness properties to determine a class of hypergraphs such that the hypergraph algebras have linear resolutions. Furthermore, nice recursive formulas for computing the Betti numbers are given.

The edge ideal of a hypergraph yields the hypergraph algebra $R / I(\mathscr{H})$.

In this way we obtain a 1-1 correspondence

\{simple hypergraphs on $[n]\}$

$\leadsto$ squarefree monomial ideals $\left.I \subseteq R=k\left[x_{1}, \ldots, x_{n}\right]\right\}$.

In Section 2 we will associate to every uniform simple hypergraph a simplicial complex, the complex of $\mathscr{H}$. Therefore, recall that an (abstract) simplicial complex on vertex set $[n]$ is a collection, $\Delta$, of subsets of $[n]$ with the property that $G \subseteq F, F \in \Delta \Rightarrow G \in \Delta$. The elements of $\Delta$ are called the faces of the complex and the maximal (under inclusion) faces are called facets. The dimension, $\operatorname{dim} F$, of a face $F$ in $\Delta$, is defined to be $|F|-1$, and the dimension of $\Delta$ is defined as $\operatorname{dim} \Delta=\max \{\operatorname{dim} F ; F \in \Delta\}$. Note that the empty set $\emptyset$ is the unique -1 dimensional face of every complex that is not the void complex \{\} which has no faces. The dimension of the void complex may be defined as $-\infty$. The $r$-skeleton of a simplicial complex $\Delta$, is the collection of faces of $\Delta$ of dimension at most $r$. Let $V \subseteq[n]$. We denote by $\Delta_{V}$ the simplicial complex

$$
\Delta_{V}=\{F \subseteq[n] ; F \in \Delta, F \subseteq V\} .
$$

For convenience, we consider 0 to be a natural number, i.e., $\mathbf{N}=\{0,1,2,3, \ldots\}$. A vector $\mathbf{j}=\left(j_{1}, \ldots, j_{n}\right) \in\{0,1\}^{n}$ is called a squarefree vector in $\mathbf{N}^{n}$. We may identify $\mathbf{j}$ with the set $V \subseteq[n]$, where $i \in V$ precisely when $j_{i}=1$. Since this correspondence between the $V$ and the $\mathbf{j}$ is bijective, we may also denote $\Delta_{V}$ by $\Delta_{\mathbf{j}}$.

If $\mathscr{H}$ is a simple hypergraph, the complex

$$
\Delta(\mathscr{H})=\{F \subseteq[n] ; E \nsubseteq F, \forall E \in \mathscr{E}(\mathscr{H})\} .
$$


is called the independence complex of $\mathscr{H}$. Note that the edges in $\mathscr{H}$ are precisely the minimal non faces in $\Delta(\mathscr{H})$. The connections between a (hyper)graph and its independence complex are explored in, for example [5], [8], [14]. In Section 2 we will see that in case of simple uniform hypergraphs $\mathscr{H}$, there is a very natural connection between the independence complex of $\mathscr{H}$ and the complex of $\mathscr{H}$.

Given a simplicial complex $\Delta$, we denote by $\mathscr{C}$.( $\Delta$ ) its reduced chain complex (see any book on algebraic topology, for example [15], for details), and by $\tilde{H}_{n}(\Delta ; k)=Z_{n}(\Delta) / B_{n}(\Delta)$ its $n$ 'th reduced homology group with coefficients in the field $k$. In general we could use an arbitrary abelian group instead of $k$, but we will only consider the case when the coefficients lie in a field. For convenience, we define the homology of the void complex to be zero.

Recall the following 1-1 correspondence, called the Stanley-Reisner correspondence:

\{simplicial complexes on $[n]\}$

sus \{squarefree monomial ideals $\left.I \subseteq R=k\left[x_{1}, \ldots, x_{n}\right]\right\}$

$$
\Delta \leftrightarrow I_{\Delta} \text {. }
$$

The ring $R / I_{\Delta}$ is called the Stanley-Reisner ring of $\Delta$. Observe that a monomial $x^{F}$ is an element in $I_{\Delta}$ precisely when $F$ is a non face in $\Delta$. By the above two 1-1 correspondences, we also get a 1-1 correspondence between the class of simple hypergraphs on $[n]$, and the class of simplicial complexes on $[n]$.

Note that the hypergraph algebra $R / I(\mathscr{H})$ is precisely the Stanley-Reisner ring of the independence complex $\Delta(\mathscr{H})$.

In Section 2, we introduce the classes of chordal and triangulated hypergraphs. The definition of triangulated hypergraph is almost identical to Definition 5.5 in [10], however, ours is more general. These classes of hypergraphs illustrate that uniform hypergraphs behave much like ordinary simple graphs. However, there are familiar properties of graphs that do not translate immediately to uniform hypergraphs. See for instance Remark 2.1 and Example 1.

It is well known, see [9], that chordal graphs are characterized by the fact that they have perfect elimination orders. We show that this remains true for hypergraphs.

In Theorem-definition 2.1 we show that the properties of being triangulated, chordal, and having a perfect elimination order, are equivalent also for hypergraphs.

In Section 4 we introduce the class of generalized chordal hypergraphs, which includes the chordal hypergraphs, and show that the corresponding hypergraph algebras, $R / I(\mathscr{H})$, have linear resolutions. Our method of proof is a natural generalization of one used by R. Fröberg in [8]. There, Fröberg 
characterizes, in terms of the complementary graphs $\mathscr{G}^{c}$, precisely for what graphs $\mathscr{G}$ the graph algebras $R / I(\mathscr{G})$ have linear resolutions. Fröberg shows:

THeOREM 1.1. Let $G$ be a simple graph on $n$ vertices. Then $k\left[x_{1}, \ldots\right.$, $\left.x_{n}\right] / I(\mathscr{G})$ has linear resolution precisely when $\mathscr{G}^{c}$ is chordal (rigid circuit, triangulated,...).

By Theorem 4.1, we obtain a partial generalization of Fröberg's theorem.

The complementary hypergraph $\mathscr{H}^{c}$, of a $d$-uniform hypergraph $\mathscr{H}$, is defined as the hypergraph on the same set of vertices as $\mathscr{H}$, and edge set

$$
\mathscr{E}\left(\mathscr{H}^{c}\right)=\{F \subseteq \mathscr{X}(\mathscr{H}) ;|F|=d, F \notin \mathscr{E}(\mathscr{H})\} .
$$

The edges of $\mathscr{H}^{c}$ may, in a natural way, be thought of as the $(d-1)$-dimensional faces in the independence complex $\Delta(\mathscr{H})$, of $\mathscr{H}$. This is how Fröberg looks at things when he proves his theorem. We show that the complex $\Delta(\mathscr{H})$ is completely determined by the edges in $\mathscr{H}^{c}$, which gives us the notion of $d$-flag complexes.

\section{The classes of chordal and triangulated hypergraphs}

In this section, all hypergraphs are assumed to be simple and uniform.

Definition 2.1. Two distinct vertices $x, y$ of a hypergraph $\mathscr{H}$ are neighbors if there is an edge $E \in \mathscr{E}(\mathscr{H})$, such that $x, y \in E$. For any vertex $x \in \mathscr{X}(\mathscr{H})$, the neighborhood of $x$, denoted $N(x)$, is the set

$$
N(x)=\{y \in \mathscr{X}(\mathscr{H}) ; y \text { is a neighbor of } x\} .
$$

If $N(x)=\emptyset, x$ is called isolated. Furthermore, we let $N[x]=N(x) \cup\{x\}$ denote the closed neighborhood of $x$.

Remark 2.1. Let $\mathscr{H}$ be a hypergraph and $V \subseteq \mathscr{X}(\mathscr{H})$. Denote by $N_{V}[x]$ the closed neighborhood of $x$ in the induced hypergraph $\mathscr{H}_{V}$. For ordinary graphs it is clear that $N_{V}[x]=N[x] \cap V$. This is not always the case for hypergraphs, as is shown in the example below. Note that the notation $N_{V}[x]$ will only occur in this remark and the example below. The fact that we do not make any greater use of it, is intimately connected to, and in a sense illustrates, the properties of the hypergraphs that we are to consider.

ExAmple 1. Consider the hypergraph $\mathscr{H}$ on vertex set $\mathscr{X}(\mathscr{H})=\{a, b, c, d$, $e\}$ and edge set $\mathscr{E}(\mathscr{H})=\{\{a, b, c\},\{a, d, e\},\{b, c, d\}\}$. Let $V=\{a, b, c, d\}$. Then $N_{V}[a]=\{a, b, c\}$ but $N[a] \cap V=\{a, b, c, d\}$.

Recall the definition of the $d$-complete hypergraph: 
Definition 2.2. The $d$-complete hypergraph, $K_{n}^{d}$, on a set of $n$ vertices, is defined by

$$
\mathscr{E}\left(K_{n}^{d}\right)=\left(\begin{array}{c}
{[n]} \\
d
\end{array}\right)
$$

where $\left(\begin{array}{l}F \\ d\end{array}\right)$ denotes the set of all subsets of $F$, of cardinality $d$. If $n<d$, we interpret $K_{n}^{d}$ as $n$ isolated points.

If $\mathscr{H}$ is a hypergraph, we associate a simplicial complex $\Delta_{\mathscr{H}}$ to it in the following way:

Definition 2.3. Given a $d$-uniform hypergraph $\mathscr{H}=(\mathscr{X}(\mathscr{H}), \mathscr{E}(\mathscr{H}))$, the complex of $\mathscr{H}, \Delta \mathscr{H}$, is the simplicial complex

$$
\Delta_{\mathscr{H}}=\left\{F \subseteq \mathscr{X}(\mathscr{H}) ;\left(\begin{array}{l}
F \\
d
\end{array}\right) \subseteq \mathscr{E}(\mathscr{H})\right\}
$$

Note that this implies that if $F \subseteq \mathscr{X}(\mathscr{H}),|F|<d$, then $F \in \Delta \mathscr{H}$.

REMARK 2.2. Note that all simplicial complexes of the form $\Delta_{\mathscr{H}}$ have complete $(d-2)$-skeleton. Two such simplicial complexes on the same vertex set thus only differ in which $(d-1)$-simplices they contain.

REMARK 2.3. Recall that a flag complex is a simplicial complex in which every minimal non face consists of precisely 2 elements. As one easily sees, such complex is determined by its 1-skeleton. According to the previous remark, $d$-flag complexes, i.e., complexes whose minimal non faces all have cardinality $d$, in a natural way generalizes flag complexes.

Proposition 2.1. $\Delta_{\mathscr{H}}=\Delta\left(\mathscr{H}^{c}\right)$, where $\Delta\left(\mathscr{H}^{c}\right)$ is the independence complex of $\mathscr{H}^{c}$.

Proof. The two complexes has the same set of vertices. $F \in \Delta\left(\mathscr{H}^{c}\right)$ precisely when $\left(\begin{array}{l}F \\ d\end{array}\right) \subseteq \mathscr{E}(\mathscr{H})$. Furthermore, $F \in \Delta\left(\mathscr{H}^{c}\right)$ for every $F \subseteq \mathscr{X}(\mathscr{H})$ with $|F|<d$.

Definition 2.4. Let $\Delta$ be a simplicial complex on a finite set, $\mathscr{X}$, of vertices. For any given $d \in \mathrm{N}$, the $d$-uniform hypergraph, $\mathscr{H}_{d}(\Delta)$, of $\Delta$, is the hypergraph with vertex set $\mathscr{X}$, and with edge set

$$
\mathscr{E}_{d}(\Delta)=\{F \in \Delta ;|F|=d\}
$$


Proposition 2.2. Let $\mathscr{H}$ be a hypergraph and $\Delta$ an arbitraryd-flag complex on $\mathscr{X}(\mathscr{H})$. Then,

- $\mathscr{H}_{d}\left(\Delta_{\mathscr{H}}\right)=\mathscr{H}$,

- $\Delta \mathscr{H}_{d}(\Delta)=\Delta$.

Proof. This follows directly from Definition 2.2 and Definition 2.3.

Definition 2.5. A hypergraph $\mathscr{H}$ is called triangulated if for every non empty subset $V \subseteq \mathscr{X}(\mathscr{H})$, either there exists a vertex $x \in V$ such that the induced hypergraph $\mathscr{H}_{N[x] \cap V}$ is isomorphic to a $d$-complete hypergraph $K_{n}^{d}$, $n \geq d$, or else the edge set of $\mathscr{H}_{V}$ is empty.

This definition is basically due to Hà and Van Tuyl, see [10] Definition 5.5. However, in [10] the property being triangulated is defined only on a special class of hypergraphs called properly-connected. For a further discussion see Section 2.2 below.

Definition 2.6. A hypergraph $\mathscr{H}$ is called triangulated $*$ if for every non empty subset $V \subseteq \mathscr{X}(\mathscr{H})$, either there exists a vertex $x \in V$ such that $N[x] \cap V$ is a facet of $\left(\Delta_{\mathscr{H}}\right)_{V}$ of dimension greater than or equal to $d-1$, or else the edge set of $\mathscr{H}_{V}$ is empty.

We will soon show (Theorem-definition 2.1) that the above two definitions are equivalent.

Definition 2.7. A chordal hypergraph is a $d$-uniform hypergraph, obtained inductively as follows:

- $K_{n}^{d}$ is a chordal hypergraph, $n, d \in \mathrm{N}$.

- If $\mathscr{G}$ is chordal, then so is $\mathscr{H}=\mathscr{G} \cup_{K_{j}^{d}} K_{i}^{d}$, for $0 \leq j<i$. (This we think of as glueing $K_{i}^{d}$ to $\mathscr{G}$ by identifying some edges, or parts of some edges, of $K_{i}^{d}$ with the corresponding part, $K_{j}^{d}$, of $\mathscr{G}_{\text {.) }}$

REMARK 2.4. For $d=2$ this specializes precisely to the class of generalized trees, i.e., generalized $n$-trees for some $n$, as defined in [8].

Remark 2.5. In the special case of simple graphs, Definition 2.5 specializes precisely to the ordinary chordal (rigid cicuit) graphs. Recall that a simple graph is called chordal if every induced cycle of length $>3$, has a chord. By considering minimal cycles, it is clear that a graph that is triangulated according to Definition 2.5, is chordal. Assume a graph $\mathscr{G}$ is chordal. It follows from Theorems 1 and 2 in [3], that the chordal graphs are precisely the generalized trees (see Remark 2.4). In a generalized tree we may easily find a vertex $x$, with the property that $\mathscr{G}_{N[x]}$ is complete, as follows: We know that $\mathscr{G}=\mathscr{G}^{\prime} \cup_{K_{j}} K_{i}$, $0 \leq j<i$. Then, we just pick a vertex $x \in \mathscr{X}\left(K_{i}\right) \backslash \mathscr{X}\left(\mathscr{G}^{\prime}\right)$, since such $x$ 
clearly has the property that $\mathscr{G}_{N[x]}$ is complete. Since every induced subgraph of a chordal graph is chordal, the same thing holds for every $\mathscr{G}_{V}, V \subseteq \mathscr{X}(\mathscr{G})$.

Another characterization of chordal graphs may be found in [9]. There it is shown that a simple graph is chordal precisely when it has a perfect elimination order. Recall that a perfect elimination order of a graph $\mathscr{G}=$ $(\mathscr{X}, \mathscr{E})$ is an ordering of its vertices, $x_{1}<x_{2}<\cdots<x_{n}$, such that for each $i, \mathscr{G}_{N\left[x_{i}\right] \cap\left\{x_{i}, x_{i+1}, \ldots, x_{n}\right\}}$ is a complete graph. The concept of perfect elimination order is well suited for generalizations. We make the following

DefinITION 2.8. A hypergraph $\mathscr{H}$ is said to have a perfect elimination order if its vertices can be ordered $x_{1}<x_{2}<\cdots<x_{n}$, such that for each $i$, either $\mathscr{H}_{N\left[x_{i}\right] \cap\left\{x_{i}, x_{i+1}, \ldots, x_{n}\right\}}$ is isomorphic to a $d$-complete hypergraph $K_{n}^{d}, n \geq d$, or else $x_{i}$ is isolated in $\mathscr{H}_{\left\{x_{i}, x_{i+1}, \ldots, x_{n}\right\}}$

Note that this specializes precisely to the definition of perfect elimination order for simple graphs if we put $d=2$.

Lemma 2.1. Let $\mathscr{H}$ be a hypergraph and $x \in V \subseteq \mathscr{X}(\mathscr{H})$ a vertex such that $\mathscr{H}_{N[x]} \cong K_{m}^{d}, m \geq d$. Then $\mathscr{H}_{N[x] \cap V}$ either is isomorphic to a d-complete hypergraph $K_{m^{\prime}}^{d}, m^{\prime} \geq d$, or else $x$ is isolated in $V$.

Proof. Either $|N[x] \cap V| \geq d$ or else $|N[x] \cap V|<d$.

REMARK 2.6. The above lemma in some sense explains what goes on in the proofs hereafter. It also casts some light on the last comment made in Remark 2.1 .

LeMma 2.2. If a hypergraph $\mathscr{H}$ with $\mathscr{E}(\mathscr{H}) \neq \emptyset$ has a perfect elimination order, then it has a perfect elimination order $x_{1}<x_{2}<\cdots<x_{n}$ in which $x_{1}$ is not isolated.

Proof. Let $x_{1}<x_{2}<\cdots<x_{n}$ be a perfect elimination order of $\mathscr{H}$, and put

$$
t=\min \left\{i ; x_{i} \text { is not isolated }\right\} .
$$

We claim that $x_{t}<\cdots<x_{n}<x_{1}<\cdots<x_{t-1}$ also is a perfect elimination order of $\mathscr{H}$. Since $x_{1}, \ldots, x_{t-1}$ are isolated, we need only verify that $\mathscr{H}_{N\left[x_{i}\right] \cap\left\{x_{i}, x_{i+1}, \ldots, x_{n}, x_{1}, \ldots, x_{t-1}\right\}} \cong K_{m_{i}}^{d}$ for some $m_{i} \geq d, i=t, \ldots, n$. However, this is clear since $\mathscr{H}_{N\left[x_{i}\right] \cap\left\{x_{i}, x_{i+1}, \ldots, x_{n}, x_{1}, \ldots, x_{t-1}\right\}}=\mathscr{H}_{N\left[x_{i}\right] \cap\left\{x_{i}, x_{i+1}, \ldots, x_{n}\right\}}$.

LemMa 2.3. If a hypergraph $\mathscr{H}$ is triangulated (triangulated*, chordal), or, has a perfect elimination order, then so does $\mathscr{H}_{V}$ for every $V \subseteq \mathscr{X}(\mathscr{H})$.

Proof. Let $V \subseteq \mathscr{X}(\mathscr{H})$. If $\mathscr{E}\left(\mathscr{H}_{V}\right)=\emptyset, \mathscr{H}_{V}$ clearly is triangulated and triangulated*. It is also chordal since we can add one vertex at a time until we 
have the desired discrete hypergraph, and any ordering of $V$ yields a perfect elimination order. Thus we may assume that $\mathscr{E}\left(\mathscr{C}_{V}\right) \neq \varnothing$.

The lemma is clear for the classes of triangulated and triangulated* hypergraphs, since if $W \subseteq V$, we have that $\left(\mathscr{H}_{V}\right)_{W}=\mathscr{H}_{W}$. Now, let $\mathscr{H}=\mathscr{G} \cup_{K_{i}^{d}} K_{i}^{d}$, $0 \leq j<i$, be chordal. If $V \subseteq \mathscr{X}(\mathscr{G})$, or if $V \subseteq \mathscr{X}\left(K_{i}^{d}\right)$, we are done by induction. If this is not the case, it is easy to realize that $\mathscr{H}_{V}=\mathscr{G}_{V} \cup_{\left(K_{j}^{d}\right)_{V}}\left(K_{i}^{d}\right)_{V}$. Since $\mathscr{G}_{V}$ is chordal by induction, the result follows. Finally, assume $\mathscr{H}$ has a perfect elimination order $x_{1}<x_{2}<\cdots<x_{n}$. Then $V$ inherits an ordering $x_{i_{1}}<x_{i_{2}}<\cdots<x_{i_{|V|}}$. The fact that this is a perfect elimination order of $\mathscr{H}_{V}$ follows from Lemma 2.1.

THEOREM-DEFINITION 2.1. Let $\mathscr{H}=(\mathscr{X}(\mathscr{H}), \mathscr{E}(\mathscr{H}))$ be a d-uniform hypergraph. Then the following are equivalent.

(i) $\mathscr{H}$ is triangulated.

(ii) $\mathscr{H}$ is triangulated*

(iii) $\mathscr{H}$ is chordal.

(iv) $\mathscr{H}$ has a perfect elimination order.

Proof. Due to Lemma 2.3, we need only consider the full set $\mathscr{X}(\mathscr{H})$ of vertices in our arguments, and we may assume that $\mathscr{E}(\mathscr{H}) \neq \emptyset$.

(i) $\Rightarrow$ (ii). Since we assume $\mathscr{E}(\mathscr{H}) \neq \emptyset$ and consider only the case where $V=\mathscr{X}(\mathscr{H})$, there is a vertex $x$ such that $\mathscr{H}_{N[x]} \cong K_{n}^{d}, n \geq d$. Then, $N[x]$ clearly is a face in $\Delta_{\mathscr{H}}$ of dimension at least $d-1$. Furthermore it has to be a facet, since if there were a $y \in \mathscr{X}(\mathscr{H}), y \neq x$, such that $N[x] \cup\{y\} \in \Delta \mathscr{H}$, then there would exist an edge $E$ with $x, y \in E$. Hence, $y \in N[x]$.

(ii) $\Rightarrow$ (i). By assumption, there is a vertex $x$ such that $N[x]$ is a facet in $\Delta \mathscr{H}$ of dimension greater than or equal to $d-1$, whence it is clear (from the definition of $\Delta_{\mathscr{H}}$ ) that $\mathscr{H}_{N[x]} \cong K_{n}^{d}$ for some $n \geq d$.

(i) $\Rightarrow$ (iii). By assumption there is a vertex $x \in \mathscr{X}(\mathscr{H})$ such that $\mathscr{H}_{N[x]} \cong$ $K_{n}^{d}$, for some $n \geq d$. Let $\mathscr{G}$ be the induced hypergraph on $\mathscr{X}(\mathscr{H}) \backslash\{x\}$. Then $\mathscr{E}(\mathscr{G})$ consists of all edges of $\mathscr{H}$, except those that contain $x$. This yields $\mathscr{H}=\mathscr{G} \cup \mathscr{K} K_{n}^{d}$, where $\mathscr{K}=K_{|N(x)|}^{d}$ on vertex set $N(x)$, and by induction we are done.

(iii) $\Rightarrow$ (i). Assume $\mathscr{H}=\mathscr{G} \cup_{K_{j}^{d}} K_{i}^{d}, 0 \leq j<i$, is chordal, where $\mathscr{G}$ is chordal by construction. If $i \geq d$, any vertex $x \in \mathscr{X}\left(K_{i}^{d}\right) \backslash \mathscr{X}(\mathscr{G})$ will do, since $\mathscr{H}_{N[x]} \cong K_{i}^{d}$ for such $x$. If $i<d$, we find, by induction, a vertex $x \in \mathscr{X}(\mathscr{G})$ with the property that $\mathscr{H}_{N[x]}=\mathscr{G}_{N[x]} \cong K_{n}^{d}$ for some $n \geq d$, since otherwise the edge set of $\mathscr{H}$ would be empty, contrary to our assumptions.

(i) $\Rightarrow$ (iv). By assumption we find a vertex $x=x_{1}$ such that $\mathscr{H}_{N\left[x_{1}\right]} \cong K_{n}^{d}$, $n \geq d$. Since the induced hypergraph on $\mathscr{X}(\mathscr{H}) \backslash\left\{x_{1}\right\}$ is triangulated, by induction it has a perfect elimination order $x_{2}<\cdots<x_{n}$. If we put $x_{1}<x_{2}$ we are done. 
(iv) $\Rightarrow$ (i). By Lemma 2.2 there is a perfect elimination order $x_{1}<\cdots<x_{n}$, such that $\mathscr{H}_{N\left[x_{1}\right] \cap V} \cong K_{m}^{d}$ for some $m \geq d$.

\subsection{Examples}

In [5], we considered hypergraph generalizations of the well known complete and complete multipartite graphs. We use these to create some examples of chordal hypergraphs.

Recall from [5] the definition of the $d$-complete bipartite hypergraph $K_{n, m}^{d}$ : This is the hypergraph on a vertex set that is a disjoint union, $[n] \sqcup[\mathrm{m}]$, of two finite sets. The edge set consists of all sets $V \subseteq[n] \sqcup[m],|V|=d$, such that $V \cap[n] \neq \emptyset \neq V \cap[m]$.

EXAmple 2. Here we consider the complement $\mathscr{H}=\left(K_{n, m}^{d}\right)^{c}$ of $K_{n, m}^{d}$. We claim that $\mathscr{H}$ is chordal. It is easy to see, considering the Stanley-Reisner ring, that $\Delta_{\mathscr{H}}$ looks like

$$
\left(\Delta_{n} \sqcup \Delta_{m}\right) \cup \Gamma_{d-2}([n] \cup[m])
$$

where $\Delta_{r}$ is the full simplex on $[r]$, and $\Gamma_{d-2}([n] \cup[m])$ is the $(d-2)$-skeleton of the full simplex on $[n] \sqcup[m]$.

Clearly, the $d$-uniform hypergraph of this complex, in other words $\mathscr{H}$, is the disjoint union two $d$-complete hypergraphs,

$$
\mathscr{H}=K_{n}^{d} \cup_{K_{0}^{d}} K_{m}^{d},
$$

so $\mathscr{H}$ is chordal.

Example 3. Now consider the complex $\Delta_{K_{n, m}^{d}}$, of $K_{n, m}^{d}$. If $n, m<d$, we have an isomorphism $K_{n, m}^{d} \cong K_{n+m}^{d}$, so in this case $K_{n, m}^{d}$ is chordal. If $n$ or $m$ is greater than or equal to $d, K_{n, m}^{d}$ is not chordal. This is because no matter which vertex $x$ we choose, the induced hypergraph on $N[x]$ cannot be $d$-complete, since it would then contain an edge lying entirely in either $[n]$ or $[m]$, which is impossible.

The general case of the $d$-complete multipartite hypergraph, $K_{n_{1}, \ldots, n_{t}}^{d}$, is similar. $K_{n_{1}, \ldots, n_{t}}^{d}$ is chordal only when $n_{i}<d$ for every $i=1, \ldots, t$. The arguments are the same as in the bipartite case.

Another kind of complete hypergraph, is the $d(a, b)$-complete hypergraph $\mathscr{H}=K_{n, m}^{d(a, b)}$, where $d=a+b, a, b \geq 1$. Here $\mathscr{X}(\mathscr{H})=[n] \sqcup[m]$, and $\mathscr{E}(\mathscr{H})=\left(\begin{array}{c}{[n]} \\ a\end{array}\right) \times\left(\begin{array}{c}{[m]} \\ b\end{array}\right)$.

Example 4. Consider the complex of $K_{n, m}^{d(a, b)}$. Pick any vertex $x$ and consider $N[x]$. If the induced hypergraph $\left(K_{n, m}^{d(a, b)}\right)_{N[x]}$ is to be complete, both $n$ 
and $m$ must be smaller than $d$, and at least one of the two equations $n=a$, $m=b$ must hold. Otherwise we obtain a contradiction since $K_{n, m}^{d(a, b)}$ would then contain an edge of the wrong shape. If $n$ and $m$ satisfy these conditions, the hypergraph is chordal.

\subsection{About being chordal, triangulated, et cetera}

The class of chordal graphs is a well studied class of graphs and indeed turns out to have many nice properties, graph-theoretical as well as algebraic. The main reason that chordal graphs behave well in so many respects is perhaps that they may be described in many equivalent ways.

In recent years several authors have generalized the properties of chordal graphs and since such generalizations may be made in many different directions, no particular standard concerning the use of the word "chordal" has been established. Thus, there is the risk of different concepts getting similar names. We comment here on a couple of interesting papers in which the concept of chordality/triangulability has been introduced.

As mentioned after Definition 2.5, the concept of triangulated hypergraph also occurs in [10]. There the authors (among other things) aim for a generalization of Fröberg's theorem (Theorem 1.1). However, the triangulated property is used on the complementary (hyper)graphs compared with how Fröberg uses it (and with how we use it). The class of triangulated hypergraphs in the sense of [10], is properly included in the class of triangulated (chordal) hypergraphs considered in this paper.

In [2] the authors (indirectly via matriods) define two classes of uniform hypergraphs, called D-perfect and triangulable respectively. It is then shown that a D-perfect hypergraph $\mathscr{H}$ is also triangulable.

It can be shown that our class of chordal hypergraphs is properly included in the class of triangulable hypergraphs in the sense of [2]. However, we do not think that the class of D-perfect hypergraphs and the class of chordal hypergraphs coincide. If our supposition holds, it can probably be proved by considering the ranks of the matroids defining the D-perfect hypergraphs. Since we are not that accustomed to matroid-theory this is a suitable topic for future research.

An algebraical aspect of the class of triangulable hypergraphs that should be mentioned, is that the definition thereof depends on the characteristic of the base field $k$. Thus we do not expect results about triangulable hypergraphs similar to Theorem-definition 2.1 or Theorem 4.1. It is however easy to show that if in a certain characteristic, $\operatorname{char}(k)$ say, a hypergraph $\mathscr{H}$ is triangulable, then the corresponding ideal $I_{\Delta \mathscr{C}}$ has linear resolution in that characteristic.

In [17] the authors note that chordal graphs may be characterized as follows: A graph $\mathscr{G}$ is chordal if and only if its vertices can be labelled by numbers in 
[n] so that $\mathscr{G}$ has no induced subgraph $\mathscr{G}_{\{i<j<k\}}$ with edges $(i, j),(i, k)$ but without the edge $(j, k)$. The authors call a graph with this property perfectly labelled. This description of chordal graphs is then used to show that (see [17], Definition 6.1, Example 6.2, Definition 9.2, and Theorem 9.4 for background) a certain kind of building sets, called graphical building sets, are chordal if and only if the underlying graph is chordal. It seems possible that chordal hypergraphs (or some variant thereof) may be connected to chordal building sets in some way, but at the present it is not clear to us how. This is another topic for further considerations. The following demonstrates that the problem is harder than it may first seem.

When first looking at Theorem 9.4 in [17], one gets the feeling that this can immediately be generalized using chordal hypergrahs. Indeed, let us make the following

Definition 2.9. A $d$-uniform hypergraph $\mathscr{H}$ on a vertex set of size $n$ is said to perfectly labelled if its vertices can be labelled by numbers in $[n]$ so that $\mathscr{H}$ has no induced subhypergraph $\mathscr{H}_{\left\{i_{1}<\cdots<i_{d+1}\right\}}$ with edges

$$
i_{1} \times\left(\begin{array}{c}
\left\{i_{2}, \ldots, i_{d+1}\right\} \\
d-1
\end{array}\right)
$$

but without the edge $\left\{i_{2}, \ldots, i_{d+1}\right\}$.

It is easy to see that this is in fact equivalent to being chordal. Thus one hopes that the proof of Theorem 9.4 in [17] goes through in an analogous hypergraph situation as well. What spoils things is the connectedness property for graphs. This property is central in the definition of graphical building set whereas for hypergraphs the notion of being connected may be defined in many different but equally natural ways (see [6] and [10] for examples). It is thus not clear how to approach the problem.

\section{Some algebraic results}

In this section we recall some results from commutative and homological algebra.

\subsection{Resolutions and Betti numbers}

To every finitely generated graded module $M$ over the polynomial ring $R=$ $k\left[x_{1}, \ldots, x_{n}\right]$, we may associate a minimal $(\mathrm{N}-)$ graded free resolution

$$
\begin{aligned}
0 \rightarrow \bigoplus_{j} R(-j)^{\beta_{l, j}(M)} \rightarrow \bigoplus_{j} R(-j)^{\beta_{l-1, j}(M)} \rightarrow & \\
\cdots & \rightarrow \bigoplus_{j} R(-j)^{\beta_{0, j}(M)} \rightarrow M \rightarrow 0
\end{aligned}
$$


where $l \leq n$ and $R(-j)$ is the $R$-module obtained by shifting the degrees of $R$ by $j$. Thus, $R(-j)$ is the graded $R$-module in which the grade $i$ component $(R(-j))_{i}$ is $R_{i-j}$.

The natural number $\beta_{i, j}(M)$ is called the $i j$ 'th $\mathrm{N}$-graded Betti number of $M$. If $M$ is multigraded we may equally well consider the $\mathrm{N}^{n}$-graded minimal free resolution and Betti numbers of $M$. The difference lies just in the fact that we now use multigraded shifts $R(-\mathbf{j})$ instead of $\mathrm{N}$-graded ones. The total $i$ 'th Betti number is $\beta_{i}(M)=\sum_{j} \beta_{i, j}$. For further details on resolutions, graded rings and Betti numbers, we refer the reader to [1], Sections 1.3 and 1.5.

The Betti numbers of $M$ occur as the dimensions of certain vector spaces over $k=R / m$, where $m$ is the unique maximal graded ideal in $R$. Accordingly, the Betti numbers in general depend on the characteristic of $k$.

A minimal free resolution of $M$ is said to be linear if for $i>0, \beta_{i, j}(M)=0$ whenever $j \neq i+d-1$ for some fixed natural number $d \geq 1$.

In connection to this we mention the Eagon-Reiner theorem.

Theorem 3.1. Let $\Delta$ be a simplicial complex and $\Delta^{*}$ its Alexander dual complex. Then $R / I_{\Delta}$ is Cohen-Macaulay if and only if $R / I_{\Delta^{*}}$ has linear minimal free resolution.

Proof. See [4], Theorem 3.

\subsection{Hochster's formula and the Mayer-Vietoris sequence}

In topology one defines Betti numbers in a somewhat different manner. Hochster's formula provides a link between these and the Betti numbers defined above.

THEOREM 3.2 (Hochster's formula). Let $R / I_{\Delta}$ be the Stanley-Reisner ring of a simplicial complex $\Delta$. The non-zero Betti numbers of $R / I_{\Delta}$ are only in squarefree degrees $\mathbf{j}$ and may be expressed as

$$
\beta_{i, \mathbf{j}}\left(R / I_{\Delta}\right)=\operatorname{dim}_{k} \tilde{H}_{|\mathbf{j}|-i-1}\left(\Delta_{\mathbf{j}} ; k\right) .
$$

Hence the total $i$ 'th Betti number may be expressed as

$$
\beta_{i}\left(R / I_{\Delta}\right)=\sum_{V \subseteq[n]} \operatorname{dim} \tilde{H}_{|V|-i-1}\left(\Delta_{V} ; k\right) .
$$

Proof. See [1], Theorem 5.5.1.

If one has $\mathrm{N}^{n}$-graded Betti numbers, it is easy to obtain the $\mathrm{N}$-graded ones via

$$
\beta_{i, j}\left(R / I_{\Delta}\right)=\sum_{\substack{\mathbf{j}^{\prime} \in \mathbb{N}^{\mathbf{n}} \\ \mathbf{j}^{\prime} \mid=\mathbf{j}}} \beta_{i, \mathbf{j}^{\prime}}\left(R / I_{\Delta}\right) .
$$


Thus,

$$
\beta_{i, j}\left(R / I_{\Delta}\right)=\sum_{\substack{V \subseteq[n] \\|V|=j}} \operatorname{dim} \tilde{H}_{|V|-i-1}\left(\Delta_{V} ; k\right)
$$

Recall that if we have an exact sequence of complexes, ${ }^{1}$

$$
\mathbf{0} \rightarrow \mathbf{L} \rightarrow \mathbf{M} \rightarrow \mathbf{N} \rightarrow \mathbf{0}
$$

there is a long exact (reduced) homology sequence associated to it

$$
\cdots \rightarrow H_{r}(N) \rightarrow H_{r-1}(L) \rightarrow H_{r-1}(M) \rightarrow H_{r-1}(N) \rightarrow \cdots
$$

When we prove Theorem 5.1, we will use this homology sequence in the special case where it is associated to a simplicial complex as follows.

Suppose we have a simplicial complex $N$ and two subcomplexes $L$ and $M$, such that $N=L \cup M$. This gives us an exact sequence of (reduced) chain complexes

$$
0 \rightarrow \mathscr{C} .(L \cap M) \rightarrow \mathscr{C} .(L) \oplus \mathscr{C} .(M) \rightarrow \mathscr{C} .(N) \rightarrow 0
$$

The non trivial maps here are defined by $x \mapsto(x,-x)$ and $(x, y) \mapsto x+y$.

The long exact (reduced) homology sequence associated to this particular sequence is called the Mayer-Vietoris sequence. More about the MayerVietoris sequence can be found in [15], Section .

\section{Generalized chordal hypergraphs}

It is easy to find an example of a uniform hypergraph $\mathscr{H}$ that is not chordal, but such that the Stanley-Reisner ring of $\Delta_{\mathscr{H}}$ has linear resolution.

EXAmPLE 5. Let $\mathscr{H}$ be the 3 -uniform hypergraph with $\mathscr{X}(\mathscr{H})=\{a, b, c, d\}$, and edge set

$$
\mathscr{E}(\mathscr{H})=\{\{a, b, c\},\{a, c, d\},\{a, b, d\}\} .
$$

The following simple picture lets us visualize $\mathscr{H}$.

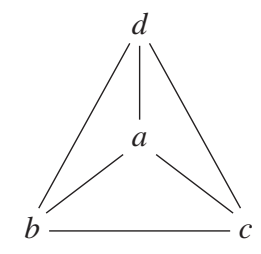

$R / I_{\Delta \mathscr{H}}$ has linear resolution, but $\mathscr{H}$ is not chordal.

\footnotetext{
${ }^{1}$ That is, complexes of modules over some ring $R$.
} 
If $\Delta$ is a simplicial complex on $[n]$ and $E$ is a finite set, we denote by $\Delta \cup E$ the simplicial complex on $[n] \cup E$ whose set of facets, $\mathscr{F}(\Delta \cup E)$, is $\mathscr{F}(\Delta) \cup\{E\}$. Similarly, if $\mathscr{H}$ is a (not necessarily $d$-uniform) hypergraph and $E$ a finite set, we denote by $\mathscr{H} \cup E$ the hypergraph on $\mathscr{X}(\mathscr{H}) \cup E$ whose edge set is $\mathscr{E}(\mathscr{H} \cup E)=\mathscr{E}(\mathscr{H}) \cup\{E\}$.

Definition 4.1. A generalized chordal hypergraph is a $d$-uniform hypergraph, obtained inductively as follows:

- $K_{n}^{d}$ is a generalized chordal hypergraph, $n, d \in \mathrm{N}$.

- If $\mathscr{G}$ is generalized chordal, then so is $\mathscr{H}=\mathscr{G} \cup{ }_{K_{j}^{d}} K_{i}^{d}$, for $0 \leq j<i$.

- If $\mathscr{G}$ is generalized chordal and $E \subseteq \mathscr{X}(\mathscr{G})$ a finite set, $|E|=d$, such that at least one element of $\left(\begin{array}{c}E \\ d-1\end{array}\right)$ is not a subset of any edge of $\mathscr{G}$, then $\mathscr{G} \cup E$ is generalized chordal.

REMARK 4.1. It is clear that every chordal hypergraph is also a generalized chordal hypergraph. Furthermore, for $d=2$ chordal graphs and generalized chordal graphs are the same.

TheOREM 4.1. Let $\mathscr{H}=(\mathscr{X}(\mathscr{H}), \mathscr{E}(\mathscr{H}))$ be a generalized chordal hypergraph and $k$ a field of arbitrary characteristic. Then the Stanley-Reisner ring of $\Delta_{\mathscr{H}}$ has linear resolution.

Proof. We consider the three instances of Definition 4.1 one at a time. If $\mathscr{H} \cong K_{n}^{d}$ we are done, since if $n \geq d$ we have a simplex so the situation is trivial, and if $n<d$ the claim is proved for example in [5], Theorem 3.1. So, we may assume $\mathscr{H} \not K_{n}^{d}$. Let $\mathscr{H}=\mathscr{G} \cup_{K_{j}^{d}} K_{i}^{d}, 0 \leq j<i$, where $\mathscr{G}$ is generalized chordal. Let $C$ and $B$ be the simplices determined by $K_{j}^{d}$ and $K_{i}^{d}$, respectively, and consider the complex $\Delta_{\mathscr{H}}^{\prime}=\Delta_{\mathscr{G}} \cup B$. Note that $B \cap \Delta \mathscr{G}=C$, $B \neq C$. We first show that $\Delta_{\mathscr{H}}^{\prime}$ has linear resolution. For every $V \subseteq \mathscr{X}(\mathscr{H})$, we have an exact sequence of chain complexes

$$
0 \rightarrow \mathscr{C} .\left(C_{V}\right) \rightarrow \mathscr{C} .\left(\left(\Delta_{\mathscr{G}}\right)_{V}\right) \oplus \mathscr{C} .\left(B_{V}\right) \rightarrow \mathscr{C} .\left(\left(\Delta_{\mathscr{C}}^{\prime}\right)_{V}\right) \rightarrow 0 .
$$

By induction, via Hochster's formula, we know that $\left(\Delta_{G}\right)_{V}$ can have non zero homology only in degree $d-2$. But then, since both $B_{V}$ and $C_{V}$ are simplices and accordingly have no homology at all, by considering the Mayer-Vietoris sequence we conclude that the only possible non zero homologies of $\left(\Delta_{\mathscr{H}}^{\prime}\right)_{V}$ lie in degree $d-2$.

Note that it is not in general true that $\Delta_{\mathscr{H}}=\Delta_{\mathscr{H}}^{\prime}$. In fact, this holds only when $d=2$. However, the difference between the two complexes is easy to understand, and we may use the somewhat easier looking $\Delta_{\mathscr{H}}^{\prime}$ to show that $\Delta_{\mathscr{C}}$ has linear resolution as well. 
To this end, let $\Gamma_{d-2}(\mathscr{X}(\mathscr{H}))$ be the $(d-2)$-skeleton of the full simplex on vertex set $\mathscr{X}(\mathscr{H})$. Then one sees that

$$
\Delta_{\mathscr{H}}=\Delta_{\mathscr{H}}^{\prime} \cup \Gamma_{d-2}(\mathscr{L}(\mathscr{H})) .
$$

The $(d-2)$-faces that we add to $\Delta_{\mathscr{H}}^{\prime}$ to obtain $\Delta_{\mathscr{H}}$, can certainly not cause any homology in degrees greater than $d-2$, that did not already exist in $\Delta_{\mathscr{H}}^{\prime}$. Indeed, suppose $\sum_{i} a_{i} \sigma_{i}$ is a cycle in a degree $r>d-2$, where $a_{i} \in k$ and the $\sigma_{i}$ 's are faces of $\Delta_{\mathscr{H}}$, of dimension $r$. Since every face $\sigma_{i}$ actually lies in $\Delta_{\mathscr{H}}^{\prime}$, it follows that $\sum_{i} a_{i} \sigma_{i}$ is a cycle also in $\Delta_{\mathscr{H}}^{\prime}$. Thus, if $\Delta_{\mathscr{H}}^{\prime}$ has linear resolution, so does $\Delta \mathscr{H}$.

Finally, let $\mathscr{H}=\mathscr{G} \cup E$. Let $F_{1}, \ldots, F_{t}$ be the elements of $\left(\begin{array}{c}E \\ d-1\end{array}\right)$ that are not subsets of any edge of $\mathscr{G}$. Note that $\Delta_{\mathscr{H}}=\Delta_{\mathscr{G}} \cup E$. Take $V \subseteq \mathscr{X}(\mathscr{H})$. If $E \nsubseteq V$, then $\left(\Delta_{\mathscr{H}}\right)_{V}=\left(\Delta_{\mathscr{G}}\right)_{V}$, so, by induction we conclude that the only possible non zero homologies of $\left(\Delta_{\mathscr{H}}\right)_{V}$ lies in degree $d-2$. Hence we may assume that $E \subseteq V$. Then we have an exact sequence

$$
0 \rightarrow \mathscr{C} .\left((\Delta \mathscr{G} \cap E)_{V}\right) \rightarrow \mathscr{C} .\left(\left(\Delta_{\mathscr{G}}\right)_{V}\right) \oplus \mathscr{C} .\left(E_{V}\right) \rightarrow \mathscr{C} .\left(\left(\Delta_{\mathscr{H}}\right)_{V}\right) \rightarrow 0 .
$$

Note that $E_{V}$ is a simplex so it has no homology, and, by induction, we know that $R / I_{\Delta \mathscr{g}}$ has linear resolution. Using Hochster's formula, we may conclude that $\tilde{H}_{d-1}\left(\left(\Delta_{G}\right)_{V} ; k\right)=0$. Hence, the Mayer-Vietoris sequence obtained from the above exact sequence looks as follows:

$$
\begin{aligned}
0 \rightarrow \tilde{H}_{d-1}\left(\left(\Delta_{\mathscr{H}}\right)_{V}\right) \rightarrow \tilde{H}_{d-2}((\Delta \mathscr{G} & \left.\cap E)_{V}\right) \\
& \rightarrow \tilde{H}_{d-2}\left(\left(\Delta_{\mathscr{G}}\right)_{V}\right) \rightarrow \tilde{H}_{d-2}\left(\left(\Delta_{\mathscr{H}}\right)_{V}\right) \rightarrow 0 .
\end{aligned}
$$

Let $z=\sum_{j} a_{j} \sigma_{j}$ be an element in $Z_{d-1}\left(\left(\Delta_{\mathscr{H}}\right)_{V}\right)$, where $\sigma_{1}=E$. Consider the expression for the derivative of this cycle

$$
0=d(z)=\cdots+\sum_{i=1}^{t} \pm a_{1} F_{i}+\cdots .
$$

Since $\sum_{i=1}^{t} \pm a_{1} F_{i}$ only can come from $d(E)$, we conclude that $a_{1}=0$. Hence $z \in Z_{d-1}\left(\left(\Delta_{G}\right)_{V}\right)$, and, using Hochster's formula, we may conclude that the Stanley-Reisner ring of $\Delta_{\mathscr{H}}$ has linear resolution.

Recall that the Alexander dual simplicial complex $\Delta^{*}$ to an arbitrary complex $\Delta$, is defined by

$$
\Delta^{*}=\{F \subseteq[n] ;[n] \backslash F \notin \Delta\} .
$$

Note that $\left(\Delta^{*}\right)^{*}=\Delta$. 
CoRollary 4.1. Let $\mathscr{H}=(\mathscr{X}(\mathscr{H}), \mathscr{E}(\mathscr{H}))$ be a generalized chordal hypergraph and $k$ a field of arbitrary characteristic. Then the Stanley-Reisner ring $R / I_{\Delta_{\mathscr{C}}^{*}}$ of the Alexander dual complex $\Delta_{\mathscr{H}}^{*}$ is Cohen-Macaulay.

PRoof. This follows by the Eagon-Reiner theorem.

Corollary 4.2. Theorem 4.1 and Corollary 4.1 in particular applies to triangulated and triangulated* hypergraphs, and also to hypergraphs that have perfect elimination orders.

REMARK 4.2. In a later work, [6], it is in fact shown that if $\mathscr{H}$ is a chordal hypergraph, then $I_{\Delta_{\mathscr{C}}}$ has linear quotients. In particular, the Alexander dual simplicial complex $\Delta_{\mathscr{H}}^{*}$ is shellable. This improves Theorem 4.1 in the case of chordal hypergraphs. It is still an open question whether this is true for generalized chordal hypergraphs as well.

Question 1. If $\mathscr{H}$ is a generalized chordal hypergraph, are there more equivalent characterizations of $\mathscr{H}$ similar to those for a chordal hypergraph given in Theorem-definition 2.1?

ACKNOWLEDGEMENTS. The author would like to thank the referee for all interesting comments and questions. They helped me improve the paper notably and have given me further topics and ideas to consider in the future.

\section{REFERENCES}

1. Bruns, W., and Herzog, J., Cohen-Macaulay Rings, revised edition, Cambridge Studies in Advanced Math. 39, Cambridge Univ. Press, Cambridge 1998.

2. Cordovil, R., Lemos, M., and Sales, L., Dirac's theorem on simplicial matroids, version 2, preprint 2007, arXiv:math/0609119.

3. Dirac, G. A., On rigid circuit graphs, Abh. Math. Sem. Univ. Hamburg 25 (1961), 71-76.

4. Eagon, J. A., and Reiner, V., Resolutions of Stanley-Reisner rings and Alexander duality, J. Pure Appl. Algebra 130 (1998), 265-275.

5. Emtander, E., Betti numbers of hypergraphs, Comm. Algebra 37 (2009), 1545-1571.

6. Emtander, E., Mohammadi, F., and Moradi, S., Some algebraic properties of hypergraphs, preprint 2008, arXiv:0812.2366.

7. Faridi, S., The facet ideal of a simplicial complex, Manuscripta Math. 109 (2002), 159-174.

8. Fröberg, R., On Stanley-Reisner rings, pp. 57-70 in: Topics in Algebra, Part 2, Proc. Warsaw 1988, Banach Center Publ. 26:2, PWN, Warsaw 1990.

9. Fulkerson, D. R., and Gross, O. A., Incidence matrices and interval graphs, Pacific J. Math. 15 (1965), 835-855.

10. Hà, H. T., and Van Tuyl, A., Monomial ideals, edge ideals of hypergraphs, and their graded Betti numbers, J. Algebraic Combin. 27 (2008), 215-245.

11. Hà, H. T., and Van Tuyl, A., Resolutions of square-free monomial ideals via facet ideals: a survey, pp. 91-117 in: Algebra, Geometry and their Interactions, Proc. Notre Dame 2005, Contemp. Math. 448, Amer. Math. Soc., Providence, RI 2007

12. Hà, H. T., and Van Tuyl, A., Splittable ideals and resolutions of monomial ideals, J. Algebra 309 (2007), 405-425. 
13. Herzog, J., and Kühl, M., On the Betti numbers of finite pure and linear resolutions, Comm. Algebra 12 (1984), 1627-1646.

14. Jacques, S., Betti Numbers of Graph Ideals, $\mathrm{PhD}$ thesis, University of Sheffield 2004, arXiv:math/0410107.

15. Maunder, C. R. F, Algebraic Topology, Dover, Mineola, NY 1996.

16. Morey, S., Reyes, E., and Villarreal, R. H., Cohen-Macaulay, shellable and unmixed clutters with a perfect matching of König type, J. Pure Appl. Algebra 212 (2008), 1770-1786.

17. Postnikov, A., Reiner, V., and Williams, L., Faces of generalized permutohedra, version 2 , preprint 2007, arXiv:math/0609184.

18. Villarreal, R. H., Cohen-Macaulay graphs, Manuscripta Math. 66 (1990), 277-293.

19. Zheng, X., Resolutions of facet ideals, Comm. Algebra 32 (2004), 2301-2324.

DEPARTMENT OF MATHEMATICS

STOCKHOLM UNIVERSITY

SE-106 91 STOCKHOLM

SWEDEN

E-mail: erice@math.su.se 